\section{Gastric-type Enteric Duplication Cyst in the Tail of a Bifid Pancreas in Association with Complete Intestinal Malrotation}

Sir,

Enteric duplication cysts (EDC) of the pancreas are rare congenital anomalies, which may be associated with relapsing pancreatitis. ${ }^{1}$ In the medical literature, a small number of reports (less than 30) have described the association of an EDC of the pancreatic tail with pancreas bifidum.2,3 Hereby, we describe a case of this dual pathology in association with complete intestinal malrotation, a unique constellation of anomalies that have not been reported previously to the best of the authors' knowledge.

A 4-year girl was brought by her parents for evaluation of severe epigastric pain and vomiting. The child was first noted to have epigastric pain at the age of 2 years, which was resolved after a few days. Gradually, these episodes increased in frequency and were sometimes associated with vomiting and aversion to food. There was no history of blood in stool, loose motions, fever or yellowish discoloration of skin. On physical examination, she had signs of mild dehydration and epigastric tenderness. Laboratory investigations revealed neutrophilic leucocytosis (white cell count of $16.4 \times 10^{9}$ cells/L with $92 \%$ neutrophils) and elevated amylase (465 U/L) and lipase $(2300 \mathrm{U} / \mathrm{L})$. She was admitted to the hospital, kept nil by mouth and administered intravenous hydration and analgesia. CT scan of abdomen with contrast was performed, which revealed the presence of a bifid pancreatic tail with an inferior moiety that was less enhancing and had surrounding fat stranding consistent with pancreatitis. Additionally, a thick-walled cyst was identified in the distal end of the inferior moiety along with radiographic signs of intestinal malrotation (Figure 1).

A diagnosis of an EDC of the tail of pancreas with a bifid pancreatic tail and superimposed pancreatitis was made. The child's abdominal pain improved gradually. She started on a soft diet and subsequently discharged home. She underwent a barium meal, which confirmed the presence of midgut malrotation. She also underwent magnetic resonance cholangiopancreatography (MRCP), which confirmed the presence of pancreas bifidum and an EDC of the tail of pancreas (Figure 2). Subsequently, she underwent exploratory laparotomy with excision of the pancreatic cyst, and Ladd's procedure for intestinal malrotation. Histopathology of the excised cyst was consistent with a gastric-type EDC of the pancreas.

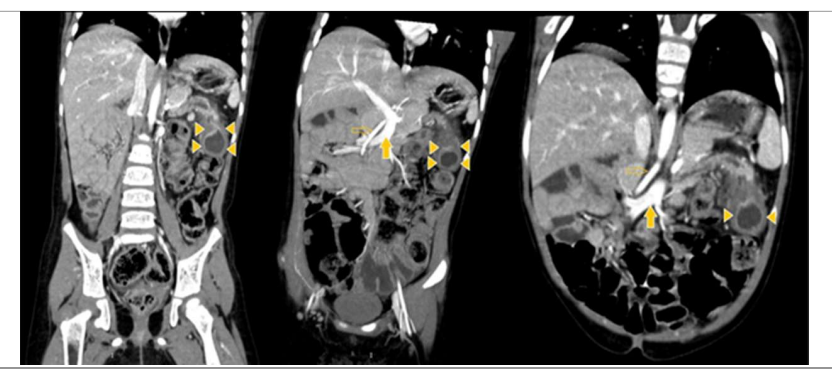

Figure 1: Coronal section of contrast-enhanced computed tomography of the abdomen demonstrating a bifid pancreatic tail with a thick-walled cyst at the distal aspect of the inferior moiety (arrowheads) along with surrounding fat-stranding. Complete intestinal malrotation is notable as evidenced by reversal of orientation of the superior mesenteric artery (solid arrow) and superior mesenteric vein (hollow arrow) along with presence of small and large bowel loops on right and left sides of abdomen, respectively.

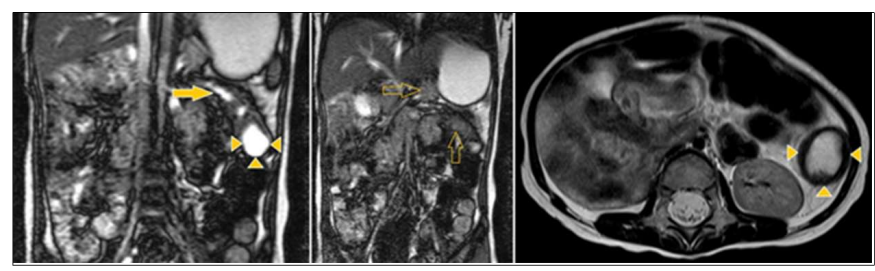

Figure 2: Magnetic resonance cholangiopancreatography demonstrating a bifid pancreas with superior and inferior moieties (hollow arrows) and an enteric duplication cyst (arrowheads) in the tail of pancreas that communicates with the inferior moiety. An irregular, dilated pancreatic duct (solid arrow) is also seen within the inferior moiety.

Patient continued to follow-up and remained healthy for six months later.

The concomitant occurrence of complete intestinal malrotation and gastric-type EDC of pancreas with pancreas bifidum can be explained by considering the embryologic process of intestinal rotation. Normal intestinal rotation is a complex process that consists of several steps, the first step of which involves $90^{\circ}$ counterclockwise rotation of the foregut (duodenal portion). ${ }^{4}$ Formation of an abnormal gastric outgrowth (duplication) adjacent to the pancreas may hamper rotation of the foregut and result in co-existent of intestinal non-rotation. From an embryologic perspective, a bifid pancreas results from aberrant branching of the main pancreatic duct and is clinically inconsequential by itself. However, when this anomaly occurs in conjunction with juxta-pancreatic enteric duplications, which communicate with the main pancreatic duct by a rudimentary duct, recurrent attacks of pancreatitis can ensue as in the present case. ${ }^{5}$ Surgical excision of the EDC can cure patients and prevent the development of pancreatic insufficiency, which may be irreversible. Paediatricians, radiologists, and paediatric surgeons need to be aware of this rare entity in order to render an accurate diagnosis and institute timely management.

\section{REFERENCES}

1. Andronikou S, Sinclair-Smith C, Millar AJ. An enteric duplication cyst of the pancreas causing abdominal pain and pancreatitis in a child. Pediatr Surg Int 2002; 18:190-2. 
2. Jain AS, Patel AM, Jain SR, Thakkar A. Accessory pancreatic lobe with gastric duplication cyst: Diagnostic challenges of a rare congenital anomaly. BMJ Case Rep 2015; pii: bcr2014207751.

3. Dinter D, Löhr JM, Neff KW. Bifid tail of the pancreas: Benign bifurcation anomaly. AJR Am J Roentgenol 2007; 189:W251-3.

4. Stringer MD. Gastrointestinal duplications. In: Puri $P$, Höllwarth $M$ (Eds). Pediatric Surgery. Heidelberg, Germany: Springer-Verlag; 2006: pp. 239-256.

5. Kapfer SA, Rappold JF. Intestinal malrotation - not just the pediatric surgeon's problem. J Am Coll Surg 2004; 199:628-35.
Muhammad Awais ${ }^{1}$, Abdul Rehman ${ }^{2}$ and Noor Ul-Ain Baloch ${ }^{2}$

1 Department of Radiology, Dow University of Health Sciences, Ojha Campus, Karachi 75270, Pakistan

2 Department of Biological and Biomedical Sciences, The Aga Khan University Hospital, Karachi 74800, Pakistan

Correspondence: Dr. Muhammad Awais, Department of Radiology, Dow University of Health Sciences,

Ojha Campus, Gulzar-e-Hijri, Suparco Road, KDA Scheme-33, Karachi 75270, Pakistan

E-mail:awaisaku@yahoo.com

Received: October 10, 2018; Accepted: November 07, 2018

.......... 